\title{
Taking complaints seriously: using the patient safety lens
}

\author{
Thomas H Gallagher, ${ }^{1}$ Kathleen M Mazor ${ }^{2}$
}

\begin{abstract}
${ }^{1}$ Department of Medicine, University of Washington, Seattle, Washington, USA ${ }^{2}$ Meyers Primary Care Institute, University of Massachusetts Medical School, Worcester, Massachusetts, USA
\end{abstract}

\section{Correspondence to} Dr Thomas H Gallagher, Department of Medicine, UW Medicine Center for Scholarship in Patient Care Quality and Safety, University of Washington, 1959 NE Pacific Street, Suite BB1240 Seattle, WA 98915 , USA; thomasg@uw.edu

Accepted 28 April 2015

\section{SLinked}

- http://dx.doi.org/10.1136/ bmjqs-2014-003834

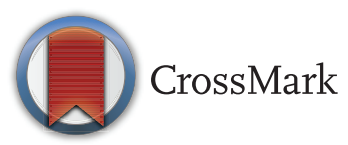

To cite: Gallagher TH Mazor KM. BMJ Qual Saf 2015;24:352-355.

\section{INTRODUCTION}

Important progress has been made towards reducing adverse events by using the modern patient safety framework, which assumes that preventable adverse events represent the combination of latent system failures and active human errors. ${ }^{1}$ This systems-oriented approach identifies provider incompetence as the root cause of a problem only after system failure and predictable human errors have been excluded. ${ }^{2}$ It relies on concepts of Just Culture and human factors-based analytic techniques to understand the aetiologies of adverse events. ${ }^{3}$ The patient safety movement prizes transparency, through early event reporting, open discussion of adverse events among colleagues and frank, empathic communication with the patient when an error has occurred. ${ }^{4}$

Yet few healthcare institutions see patient complaints as adverse events. Instead, at most institutions, patient complaints are handled by patient relations or risk management departments, with a primary goal of mollifying the patient and avoiding litigation, missing the opportunity not only to meet the affected patients' needs but also to improve the quality of care going forward by identifying root causes and developing prevention plans.

It should come as no surprise that we do not take patient complaints as seriously as we do critical incidents such as wrong site surgery. It is easy to dismiss complaints as attributable to the provider's interpersonal skills or the patient's personality. However, the thoughtful paper by Spittal and colleagues ${ }^{5}$ reminds us that like any adverse event, patient complaints have an epidemiology that can yield important lessons for prevention. To optimise learning and prevent future complaints, we need to fully apply the patient safety lens and systematically examine the multiple causes contributing to these complaints.
Spittal and colleagues build on their prior publication that found patient complaints against Australian physicians were highly clustered: half of all complaints were generated by only $3 \%$ of physicians in the study. ${ }^{6}$ The current paper extends these findings and describes the Predicted Risk of a New Event (PRONE) score to identify physicians at risk of recurrent complaints. The potential utility of the PRONE score is clear: it could move the healthcare institutions, regulators and liability insurers who field patient complaints from a reactive to a proactive posture. By identifying complaint-prone physicians and providing prospective remediation, patient dissatisfaction, quality of care problems and litigation risk associated with complaint-prone providers could all be reduced. The ability to target at-risk providers, and thereby efficiently use limited and expensive resources -including provider time-is clearly attractive. Limited self-awareness by providers is a common root cause of recurrent patient complaints. An empirically derived score indicating the recipient is at high risk of recurrent complaints may be hard to ignore and could motivate an at-risk provider to pursue improvement. ${ }^{7}$

The PRONE score concept and results also raise important questions. While the authors made efforts to incorporate the nature of the complaint into their analysis, ultimately the PRONE score treats all complaints equally. In reality, complaints are heterogeneous, stimulated by diverse events and circumstances, with differing degrees and types of harm. ${ }^{8}$ We learn from the authors' analysis that certain specialties are at much greater risk of complaints, with plastic surgeons and dermatologists having a fourfold higher risk of complaints compared with anaesthesiologists and radiologists. But we do not know what proportion of the complaints against plastic surgeons and dermatologists represented patient dissatisfaction with the outcome of a cosmetic procedure, dissatisfaction 
potentially exacerbated by having paid for the procedure out of pocket. The appropriate response to a provider whose elevated PRONE score is attributable to expectations about surgical outcomes is likely to differ from the response to a provider who generates repeated complaints about serious delays in diagnosis. Clearly, a 'one size fits all' approach will not work. As the authors note, information such as the details of the actual complaints is needed to understand why an individual provider is at risk and what steps could prevent recurrences.

\section{TAKING COMPLAINTS SERIOUSLY}

Until recently, the patient perspective has been heavily discounted in favour of the provider perspective, which is largely medical. But patients and family members can provide unique and important insights, especially around care breakdowns. ${ }^{9}{ }^{10}$ When care is complex, fragmented or rife with transitions, patients and family members may be the only ones aware of how care is actually delivered. In many situations, patients and family members may be the first to detect lapses in safety or quality, identify worsening outcomes or point out breakdowns in communication that providers have missed.

The fact that complaints are relatively rare contributes to the tendency to undervalue them. Even for the most complaint-prone provider, the number of complaints relative to the total number of patients seen is extremely small. Small numbers can be interpreted to mean that the 'complainers' are unusual, and that their perceptions are wrong or at least substantively different from other patients' perceptions, making it easy for a provider to believe that there is no need to change. While leaders charged with improving care quality and patients' experiences may recognise that a small number of complaints can signal a significant problem, the small number of events may make it difficult to identify underlying problems with confidence.

Context is always critical when interpreting data, but is especially so for understanding complaints. The PRONE score provides one key piece of contextual information, that is, whether a given complaint is best interpreted as an isolated event or a sign of future problems. There is, however, a need to look deeper in order to identify recurrent problems and patterns within and between providers, specialties and systems. Complaints could be examined along multiple dimensions, such as provider behaviour (eg, rudeness, insufficient information provision, insensitive delivery of a diagnosis), clinical situations (eg, decision making about surgery, transitioning to palliative care) as well as the more obvious dimensions such as patient diagnoses. Identification of patterns of complaints would inform interventions. For instance, if complaints about providers not listening were particularly common in certain clinical situations, providers who face those situations routinely could be offered targeted training around listening skills.

\section{THE PATIENT SAFETY LENS}

The lens through which a patient complaint is viewed can reduce or enhance its information value. A risk management lens focuses on liability and the potential for financial or reputational harm to the organisation and the individual provider. Such a focus will necessarily miss opportunities to learn and improve care.

A more useful lens-one that would favour learning over protection-is the patient safety lens. A patient safety lens would compel organisations to treat patient complaints as adverse events, triggering efforts to mitigate any harm to the patients, as well as systematic and systemic efforts to prevent recurrences. Such an approach could drive system improvements that would truly matter to patients. Patients who file complaints believe that they have been harmed. The harm may not be physical-it may be emotional distress, life disruption or loss of trust. Regardless, the harm is experienced as significant and damaging, and the patient wants it to be taken seriously. Healthcare systems and providers need to respond accordingly, shifting their focus from efforts to mollify and avert publicity to identifying root causes and improving care; the patient safety lens has the potential to help with this shift in focus. Applying this lens to complaints would certainly increase the number and type of events that would be considered and might also lead to a greater appreciation of the patient's perspective on harms.

Critical components of applying the patient safety lens to complaints would include:

1. Using the Just Culture and human factors approach. Understanding complaints as adverse events would reinforce the importance of applying the same Just Culture and human factors perspectives to complaints that are used for any adverse event. ${ }^{11}$ In situations where the complaint originated from system failure or human error, the provider would be consoled. For those complaints that were rooted in at-risk behaviour, coaching would be indicated. And punitive action would be reserved for complaints that represented recklessness or deliberate insensitivity on the part of the provider. Implicit in this application of the Just Culture and human factors paradigms would be the use of formal event analysis for at least a portion of patient complaints to identify the fundamental root causes and develop systembased prevention plans, such as communication skills training or revisions to complaint-prone care delivery processes. $^{12}$

Yet the paper by Spittal and colleagues, which focuses on the issue of recurrent complaints, exposes a potential weak spot in the application of Just Culture concepts: at what point do recurrent complaints, even in the absence of obvious reckless behaviour on the part of the provider, cross over into requiring disciplinary action? There is a growing body 
of literature on the predictors of unprofessional and disruptive providers which underscores the need to take a systematic approach to both collecting and acting on such data. ${ }^{13}$ If providers are generating multiple complaints through what could be construed as at-risk behaviour, especially in the face of feedback and coaching about how to modify the at-risk behaviour, this pattern itself represents recklessness that would merit disciplinary action. ${ }^{14}$

2. Better reporting systems for identifying and responding to patient complaints are required. Cumbersome, difficult-to-use reporting systems may discourage complaints and are suboptimal because they expose only the tip of the iceberg. ${ }^{10}$ For every complaint received, an unknown number of patients has had similar experiences and suffered similar harm in silence. Proactive surveillance coupled with simple, easy to activate reporting systems would provide more complete information and lead to greater learning, much as improved adverse event reporting has been enabled patient safety efforts. Strategies for rapidly categorising complaints would further enhance the value of increased reporting. ${ }^{8}$ Of course, systems which actively encourage complaints will need to include effective strategies for responding to problems, or dissatisfaction will only be exacerbated.

3. Transparency and open communication is key. Transparency in all its forms is critical to detecting and responding to the care breakdowns that generate patient complaints, just as such openness is essential to addressing any patient safety problem. Once the care breakdown has been identified, transparent and open communication with the affected patient, following current recommendations for disclosure after any adverse event, is critical. $^{15}$ Post-complaint conversations should include transparency about what occurred, plans to prevent recurrences, a sincere apology and acknowledgement of the patient's experience of harm. ${ }^{16}$ And, the profession is going to need to become more transparent with the public (at least in summary form) about patient complaints, including how these concerns are being addressed if public trust in the profession's commitment to improving care is to be restored.

4. Patient safety science is early in its development, and more research is needed. Perhaps the most important question that arises from the findings of Spittal and colleagues is how to intervene when patterns of recurrent complaints are detected. The PRONE score focuses on recurrences for a specific provider, but complaints could also be evaluated for recurrence within healthcare systems or clinical situations. These different perspectives might help to determine whether interventions are best focused on individuals, organisations or both.

5. Be wary of simplistic solutions. The patient safety movement provides a cautionary tale regarding the challenges of preventing adverse events, reminding us that reducing complaints involves culture change, and so will require patience, time and resources. Experts in high reliability organisations also emphasise the need to avoid overly simplistic conceptualisations of the problem or potential solutions.
New systems at the institutional and regulatory level are needed for monitoring and responding to patient complaints. One important challenge will be to identify the most appropriate roles for different stakeholders. Healthcare organisations have pertinent local information about why a provider may be generating complaints but also potentially have the strongest incentive to ignore such problems. External regulators such as state boards of medicine possess the public accountability and ability to respond objectively to complaints that healthcare institutions may lack and also have powerful tools for motivating physicians to change their behaviour such as taking disciplinary action against physicians' licences. But these external regulators often have little access to data about the overall performance of the provider in question, peer review information that healthcare institutions guard zealously.

The patient safety movement has taught us that progress in reducing adverse events begins with taking preventable harm seriously, and goes on to stress the value of a systems perspective to understand the contributing factors and to design innovative error reduction strategies. Progress in reducing patient complaints will require the same approach, and must also begin with a change in perspective. The patient safety movement challenged the belief that complications were 'the cost of doing business' and highlighted that many harms, from adverse drug events and healthcareassociated infections through to procedural complications and missed diagnoses, could be avoided. Similarly, we must overcome the inclination to discount patient complaints. Analysing complaints systematically and recognising situations and providers likely to generate complaints constitute crucial steps in designing strategies to reduce complaints. But the first and most important step entails expanding our perspective beyond the technical execution of care to encompass and appreciate patients' reports of their care experiences.

Funding Agency for Healthcare Research and Quality Grant \#5R18HS022757-02.

Competing interests None declared.

Provenance and peer review Not commissioned; internally peer reviewed.

\section{REFERENCES}

1 Institute of Medicine (U.S.). Committee on Quality of Health Care in America. In: Crossing the quality chasm: a new health system for the 21st century. Washington DC: National Academy Press, 2001.

2 Wachter RM. Understanding patient safety. New York: The McGraw-Hill Companies, Inc., 2012.

3 Marx D. Patient safety and the "Just Culture": a primer for health care executives. New York: Columbia University, 2001.

4 Bell SK, White AA, Yi JC, et al. Transparency when things go wrong: physician attitudes about reporting medical errors to patients, peers, and institutions. J Patient Saf 2015. 
5 Spittal M, Bismark M, Studdert D. The PRONE score: an algorithm for predicting doctors' risks of formal patient complaints using routinely collected administrative data. $B M$ J Quality Saf 2015;24:360-8.

6 Bismark MM, Spittal MJ, Gurrin LC, et al. Identification of doctors at risk of recurrent complaints: a national study of healthcare complaints in Australia. BMJ Qual Saf 2013;22:532-40.

7 Hickson GB, Entman SS. Physician practice behavior and litigation risk: evidence and opportunity. Clin Obstet Gynecol 2008;51:688-99.

8 Reader TW, Gillespie A, Roberts J. Patient complaints in healthcare systems: a systematic review and coding taxonomy. BMJ Qual Saf 2014;23:678-89.

9 Weissman JS, Schneider EC, Weingart SN, et al. Comparing patient-reported hospital adverse events with medical record review: do patients know something that hospitals do not? Ann Intern Med 2008;149:100-8.
10 Mazor KM, Roblin DW, Greene SM, et al. Toward patientcentered cancer care: patient perceptions of problematic events, impact, and response. J Clin Oncol 2012;30:1784-90.

11 Connor M, Duncombe D, Barclay E, et al. Creating a fair and just culture: one institution's pat toward organizational change. Jt Comm J Qual Patient Saf 2007;33:617-24.

12 Levinson W, Pizzo PA. Patient-physician communication: it's about time. JAMA 2011;305:1802-3.

13 Shojania KG, Dixon-Woods M. 'Bad apples': time to redefine as a type of systems problem? BMJ Qual Saf 2013;22:528-31.

14 Lesser CS, Lucey CR, Egener B, et al. A behavioral and systems view of professionalism. JAMA 2010;304:2732-7.

15 Truog RD, Browning DM, Johnson JA, et al. Talking with patients and families about medical error. Baltimore: The Johns Hopkins University Press, 2011.

16 Mazor KM, Greene SM, Roblin D, et al. More than words: patients' views on apology and disclosure when things go wrong in cancer care. Patient Educ Couns 2013;90:341-6. 